\title{
Bacterial Urinary Tract Infections among Males with Lower Urinary Tract Obstruction at Komfo Anokye Teaching Hospital, Kumasi, Ghana*
}

\author{
Christian Kofi Gyasi-Sarpong ${ }^{1 \#}$, Edwin Mwintiereh Ta-ang Yenli ${ }^{1}$, Ali Idriss ${ }^{2}$, Addae Appiah Arhin ${ }^{1}$, \\ Ken Aboah ${ }^{1}$, Roland Azorliade ${ }^{1}$, Yaw Agyekum Boaitey ${ }^{3}$, Augustina Angelina Annan ${ }^{4}$ \\ ${ }^{1}$ Department of Surgery (Urology Unit), Komfo Anokye Teaching Hospital, Kumasi, Ghana \\ ${ }^{2}$ Microbiology Department (Agogo), Kumasi Centre for Collaborative Research in Tropical Medicine, Kumasi, Ghana \\ ${ }^{3}$ Microbiology Department, Komfo Anokye Teaching Hospital (KATH), Kumasi, Ghana \\ ${ }^{4}$ Data Management Department, Kumasi Centre for Collaborative Research in Tropical Medicine (KCCR), Kumasi, Ghana \\ Email: "gaysek@yahoo.com, mwintyus@yahoo.com, drissli101@yahoo.com, addaiarhin@yahoo.com, surgksi@yahoo.com, \\ azor72@yahoo.com, yawboaitey30@yahoo.com, auguean@yahoo.com
}

Received April 21, 2012; revised May 28, 2012; accepted June 10, 2012

\begin{abstract}
Purpose: We describe the commonest pathology responsible for lower urinary tract obstruction (LUTO) and associated symptoms such as UTI, etc. among males at the Komfo Anokye Teaching Hospital (KATH) in Kumasi. Materials and Methods: Between January and December 2009, prospective cross sectional hospital based study was conducted involving 103 subjects. Patients with symptoms of LUTO and who were on short admission (up to 48 hours) at the accident and emergency unit of KATH were identified as potential study subjects. All the patients presented with acute retention of urine at the emergency unit of KATH and urethral catheterization was attempted to relieve them of the retention under sterile conditions. Those patients whose initial catheterization failed went through suprapubic cystostomy (suprapubic catheterization). Urine specimens for culture and sensitivity tests were then collected into sterile urine containers immediately after the catheterization (irrespective of type). Ultrasound Scan was requested for all the patients to assist in diagnosing enlargement of the prostate and other associated urological pathologies. Retrograde urethrogram was however, requested for those patients with suprapubic catheterization and which helped in the diagnosis of the urethral stricture. For a particular child, urethral catheter was passed under sterile condition and Micturicting Cysto-Urethrogram (MCUG) was done to assist in diagnosing posterior urethral valve and other conditions. Those subjects whose urinalysis revealed pyuria and positive urine culture and who had voluntarily agreed to enter the study were enrolled. Subjects whose urine culture had multiple bacteria growth that was deemed to be contaminants were excluded from the study. Results: The mean age for males with LUTO was 62 years. The youngest male with LUTO was aged 2 years whilst the maximum age was 93 years. LUTO due to prostatic hyperplasia was found in $79(76.7 \%)$ men; $23(22.3 \%)$ had LUTO due to urethral stricture and $1(1.0 \%)$ (youngest male). The mean age of patients that had LUTO due to urethral stricture was approximately 39 years. The youngest patient with LUTO due to urethral stricture aged 22 years whilst the maximum age was 72 years. Microbiological results indicated that Escherichia coli caused $53(51.5 \%)$ of urinary tract infection (UTI) due to LUTO; Klebsiella $=23$ (22.3\%); Staphylococcus aureus $=14$ (14.6\%); Pseudomonas spp. $=8(7.8 \%)$ Proteus $=2(2.0 \%)$; Citrobacter $=1(1.0 \%)$ and Salmonella spp. $=1(1.0 \%)$. Conclusion: The most common cause of lower urinary tract obstruction (LUTO) in Kumasi, Ghana is prostatic hyperplasia and the commonest causative organism of the associated UTI symptom is Escherichia coli.
\end{abstract}

Keywords: Hyperplasia; Prostatic; Dysuria; Micturition; Nocturia; Suprapubic; Haematuria; Nephelometer; Asymptomatic; Bacteriuria; Acute; Cystitis; Prostatitis; Pyelonephritis; Osteomyelitis; Urosepsis; Cystoscopy; Catheter; Benign; Stricture

\section{Background}

With advancing age, the lower urinary tract becomes an increasingly important source of medical and social con-

\footnotetext{
"Competing interests: None declared.

\#Corresponding author.
}

cern. Lower urinary tract obstruction (LUTO) (also known as Bladder outlet obstruction (BOO)), urinary tract infections (UTIs), bladder cancer, incontinence, etc. are all more common in elderly patients. Although, advancement in medical care has contributed greatly to the understanding of the pathogenesis, diagnosis and treat- 
ment of bladder outlet obstruction (BOO), the disease still remains a major clinical problem in most developing countries including Ghana.

Lower urinary tract symptoms (LUTS) are common in elderly men and have a negative impact on quality of life. With the understanding of the pathophysiology of male voiding disorders, it is known that not only the prostate but also the urinary bladder is responsible for LUTS. $\mathrm{BOO}$ often produces lower urinary tract symptoms. By definition, BOO is determined by urodynamic studies assessing the pressure-flow relationship during voiding. Since the 1960s, much study has been done to standardize the urodynamic definition of obstruction in men. Currently, pressure-flow studies remain the gold standard for the diagnosis of BOO and the aetiology of LUTS [1].

Although bladder outlet obstruction can occur in both women and men, the condition is most common in older men. It occurs at the base of the bladder when the opening between the bladder and urethra is partially or completely blocked. The condition therefore reduces or prevents urine from emptying from the bladder.

The causes of BOO vary between the sexes. The most common cause in men (present in as many as $70 \%$ of men older than 70 years) is noncancerous enlargement of the prostate, which is also known as benign prostatic hyperplasia (BPH). Bladder outlet obstruction can also be caused by prostate cancer. Other causes of bladder outlet obstruction that occur frequently in men include urethral stricture, bladder diverticula, bladder stones, bladder tumors, etc. Urethral stricture patients most often present with symptoms of obstruction and usually have a history of urethral trauma, surgery, instrumentation, or sexually transmitted diseases $[2,3]$.

Symptoms of BOO include: urinary tract infection (UTI), urinary hesitancy, acute urinary retention, urinary intermittency, weak urine stream, frequent urination, abdominal pain, pain during urination, etc.

UTI is an inflammatory response of the urothelium to bacterial invasion that is usually associated with bacteriuria and pyuria [4]. The gold standard for the diagnosis of UTI is positive urine culture. A bacteria colony count of $\geq 10^{5}$ colony forming units (CFU) per milliliter (ml) of midstream urine has classically been used as the culture-based definition of UTI [5]. But, colony count of at least $10^{2}$ $\mathrm{CFU} / \mathrm{ml}$ is diagnostic for UTI if the specimen is obtained from catheterized patient or suprapubic aspiration [6,7].

The prevalence of UTI increases with age and is twice as high in women as in men [8,9]. There are unique risk factors for the development of UTI among men, which include urinary stasis from bladder outlet obstruction (BOO) due to benign prostatic hyperplasia, instrumentation such as cystoscopy and catheterization [10]. UTI may be asymptomatic or symptomatic. The symptoms of UTI are dysuria, urgency and frequency of micturition, nocturia, suprapubic pain, haematuria, flank pain, fever and chills. Patients with indwelling catheter may be asymptomatic [4].

In the United States, the top five aetiologic gram negative organisms for UTI in descending order of frequency include: Escherichia coli (E. coli), Pseudomonas aeruginosa, Klebsiella species (spp.), Enterobacter species and A. baumannii [11]. In Nigeria the top five aetiologic agents for catheter associated UTI in descending order are Klebsiella spp., Pseudomonas spp., Escherichia coli, Staphylococcus aureus and Proteus mirabilis [12]. Among pregnant women attending antenatal clinic at Komfo Anokye Teaching Hospital (KATH) Kumasi, Ghana, E. coli and Staphylococcus aureus are the leading causes of asymptomatic bacteriuria [13]. The complications of UTI may range from acute cystitis, prostatitis, pyelonephritis, osteomyelitis to urosepsis [10].

Many aspects of BOO in males and associated symptoms such as UTI are unclear or poorly described. To the best of our knowledge the causes of $\mathrm{BOO}$ in males with associated symptoms like the urinary tract infections at KATH, Kumasi, Ghana has not yet been reported. Thus, the generalized concept of $\mathrm{BOO}$ from other parts of Africa and the Western population may not hold true for Ghana. This study was therefore set out to establish the commonest pathology precipitating BOO and the associated symptoms at KATH, Kumasi, Ghana.

\section{Materials and Methods}

\subsection{Subjects}

A prospective cross sectional hospital based study was conducted involving 103 subjects from January to December 2009. Patients with symptoms of BOO and who were on short admission (up to 48 hours) at the accident and emergency unit of KATH were identified as potential study subjects.

All the patients presented with acute retention of urine at the emergency unit of KATH and urethral catheterization was attempted to relieve them of the retention under sterile conditions. A number of those patients whose initial catheterization failed went through suprapubic cystostomy (suprapubic catheterization). Urine specimens for culture and sensitivity tests were then collected into sterile urine containers immediately after the catheterization (irrespective of type).

\subsection{Procedures}

Ultrasound Scan was requested for all the patients to assist in diagnosing enlargement of the prostate and other associated urological pathologies. Retrograde urethrogram was however, requested for those patients with suprapubic catheterization and which helped in the diagno- 
sis of the urethral stricture. For a baby, urethral catheter was passed under sterile condition. Micturicting Cystorethrogram (MCUG) was done to assist in diagnosing posterior urethral valve and other conditions. The patients were then discharged and reported to the urologist at the outpatient department (urology department) after three days with their microbiology and imaging reports where available.

Those subjects whose urinalysis revealed pyuria and positive urine culture and who had voluntarily agreed to enter the study were enrolled. Subjects whose urine culture had multiple bacteria growth that was deemed to be contaminants were excluded from the study.

\subsection{Specimen Collection}

All the patients presented with acute retention of urine at the accident and emergency unit of KATH and urethral catheterization was attempted to relieve them of the retention under sterile conditions. A number of those patients whose initial catheterization failed went through suprapubic cystostomy (suprapubic catheterization). The importance of clean catch urine specimen was explained to consented study subjects. About $10 \mathrm{ml}$ of urine specimens for culture and sensitivity tests were then collected from the distal end of the catheter immediately after the catheterization (irrespective of type) into sterile urine containers. Collected urine specimens were transported immediately to the medical microbiology laboratory of KATH for microscopy, culture and sensitivity.

\subsection{Microscopy and Culture}

The urine specimens received at the medical microbiallogy laboratory of KATH were processed within 2 hours. The specimens on receipt were divided appropriately for culture and sensitivity as well as microscopy.

\subsection{Urine Microscopy}

$5 \mathrm{ml}$ of the urine specimens for routine examination was aseptically transferred into centrifuge tubes and spun at 1000 revolutions for 5 minutes. Each supernatant decanted and the sediment remixed by tapping the bottom of the tube. One drop of well mixed sediment was transferred to a microscopic slide, cover-slipped and examined microscopically using the $10 \times$ and $40 \times$ objective lens with condenser iris closed sufficiently [14]. The number of pus cells per high power field was then recorded for each specimen. Urine specimens having pus cells $>5$ per high field were cultured whilst those with pus cells $<5$ per high field were ignored.

\subsection{Urine Culture}

The urine specimens were gently mixed to avoid foaming.
A calibrated loop $(1 \mu \mathrm{l})$ full of the unspun urine was inoculated onto a CLED agar and streaked out for single colonies. These were incubated under aerobic conditions $\left(35^{\circ} \mathrm{C}-37^{\circ} \mathrm{C}\right)$ for $16-24 \mathrm{~h}$. One plate was used for each specimen and correctly labeled. The plates were examined after the incubation period for significant growth. Gram stain, motility test and biochemical tests such as Kliglier, citrate, urea, ornithine, indole, oxidase, as well as coagulase tests were performed to assist in the identification of the bacterial pathogens. Confirmations of the identified organisms were done using Becton Dickinson's Phoenix Spec Nephelometer and $\mathrm{BBL}^{\mathrm{TM}} \mathrm{Crystal}^{\mathrm{TM}}$ semiauto reader for bacterial pathogens at Kumasi Centre for Collaborative Research in Tropical Medicine (Microbiology Laboratory, Malaria Research Centre, Agogo).

All the microbiology procedures were quality controlled using American Type Culture Collection (ATCC) strains.

\subsection{Bio-Data Analysis}

Data entry sheet was used to record subject's sociodemographic characteristics, underlying cause of BOO and urine culture results. Data was then captured on epidata version 3.0 for cleaning and validation. Data was analysed using SPSS 16.0 statistical package.

\section{Results}

All the study subjects were in patients who were on short admissions (up to 48 hours) at the accident and emergency unit of KATH as described at the methods section above. Our study showed that patients with lower UTI and LUTO came from varied occupational background. The predominant occupation was farming $(\mathrm{n}=30)$, followed by pensioners $(n=17)$, traders $(n=13)$, civil servants $(11)$, teachers $(n=9)$, drivers $(n=5)$, security personnel $(\mathrm{n}=4)$, mechanic $(\mathrm{n}=3)$, pastors $(\mathrm{n}=2)$, students $(\mathrm{n}=2)$, nurse $(\mathrm{n}=1)$, carpenter $(\mathrm{n}=1)$, baby $(\mathrm{n}=1)$, goldsmith $(\mathrm{n}=1)$ and unemployed $(\mathrm{n}=3)$.

The ultrasound scan results revealed enlargement of the prostate for 79 out of $103(76.7 \%)$ subjects. Retrograde urethrogram requested for those with suprapubic catheterization also revealed $23(22.3 \%)$ of the subjects having urethral stricture whereas Micturicting CystoUrethrogram (MCUC) also helped in diagnosing 1 (1.0\%) subject having posterior urethral valve.

The mean age for men with LUTO was 62 years. The youngest male with LUTO was aged 2 whilst the maximum age was 93 years. LUTO due to prostatic hyperplasia was found in $79(76.7 \%)$ of the patients. $23(22.3 \%)$ of the patients had LUTO due to urethral stricture and only $1.0 \%$ had posterior urethral valve as the cause of LUTO as in Table 1 below. The youngest male's obstruction was due to posterior urethral valve. The mean 
Table 1. Causes of LUTO among males in KATH, Kumasi, Ghana $(n=103)$.

\begin{tabular}{ccc}
\hline Disease entity & Frequency & Percentages \\
\hline Prostatic hyperplasia & 79 & 76.7 \\
Urethral stricture & 23 & 22.3 \\
Posterior urethral valve & 1 & 1.0 \\
Total & 103 & 100.0 \\
\hline
\end{tabular}

age of patients that had LUTO due to urethral stricture was approximately 39 years. The youngest patient with LUTO due to urethral stricture aged 22 years whilst the maximum age was 72 years.

Microbiological laboratory results indicated that $E$. coli caused $51.5 \%$ of lower UTI; $23.3 \%$ by Klebsiella; $14 \%$ by Staphylococcus aureus as well as $8 \%$ by Pseudomonas spp. Finally, the contributions of Proteus, Citrobacter, and Salmonella spp. for the lower UTI were $2 \%, 1 \%$ and $1 \%$ respectively.

\section{Discussion}

With advancing age, the lower urinary tract becomes an increasingly important source of medical and social concern. Lower urinary tract symptoms (LUTS) are common in elderly men and have a negative impact on quality of life. With the understanding of the pathophysiology of male voiding disorders, it is known that not only the prostate but also the urinary bladder is responsible for LUTS. BOO has been identified to be a common cause of LUTS. By definition, BOO is determined by urodynamic studies assessing the pressure-flow relationship during voiding [1].

Although bladder outlet obstruction can occur in both women and men, the condition is most common in older men. It occurs at the base of the bladder when the opening between the bladder and urethra is partially or completely blocked thereby resulting in reducing or preventing urine emptying from the bladder.

The causes of $\mathrm{BOO}$ vary between the sexes. The most common cause in men (present in as many as $70 \%$ of men older than 70 years) is noncancerous enlargement of the prostate, which is also known as benign prostatic hyperplasia (BPH). Other causes of bladder outlet obstruction that occur frequently in men include prostate cancer, urethral stricture, bladder diverticula, bladder stones, bladder tumors, etc. Urethral stricture patients most often present with symptoms of obstruction and usually have a history of urethral trauma, surgery, instrumentation, or sexually transmitted diseases $[2,3]$.

Urinary tract infections (UTIs) are a group of infections of the urinary tract. The most common UTI is cystitis. Other UTIs involve the urethra (urethritis) or kidneys (pyelonephritis). UTI may be caused by bacteria, an ob- struction in the flow of urine (such as a large kidney stone or enlarged prostate gland in men), an indwelling urinary catheter (IDC) as well as sexual intercourse, especially in women. UTI accounts for over 7 million visits to physicians' offices and necessitates or complicate over one million hospital admissions in the United States yearly [15-17]. Clinically, urinalysis that reveals both bacteriuria and pyuria is diagnostic of UTI [6]. The gold standard for the diagnosis of UTI is a positive urine culture. This study was therefore designed to establish the commonest pathology precipitating BOO (LUTO) and the associated symptoms at KATH, Kumasi, Ghana.

In this study, 79 out of 103 subjects $(76.7 \%)$ were diagnosed of prostatic hyperplasia as the cause of LUTO through ultrasonography. Based on this finding, there is ample evidence that suggests that prostatic hyperplasia could be a major probable cause of LUTO (BOO) among males residing in the Kumasi metropolis of Ghana. This result however contradicts the findings of a study conducted by Kuo (1999) [18] that reported smaller prostate sizes in Taiwanese men despite having similar age group as well as clinical symptoms of moderate to severe LUTO (BOO) but different imaging equipment. Generally, $\mathrm{BOO}$ is an urodynamic condition implying voiding with high pressure and/or low flow rate.

Racial, ethnic and genetic variation could most probably account for the differences in prostate size. Some data also suggest that prostatic hyperplasia arises due to a defect in the stromal control component within the unique stromal-epithelial interaction [19].

Another important factor in the development of hyperplastic tissue within the prostate may be interaction between local growth factors and steroid hormones. These growth factors include a diverse stimulatory group such as insulin-like growth factor (IGF), transforming growth factor-beta (TGF- $\beta$ ) and other epithelial type growth factors [20]. However, one limitation of this study was the inability to further differentiate the benign cases from the malignant ones due to resource constraints.

The present study also recorded $22.3 \%$ (23 out of 103 ) of the study population having urethral stricture (diagnosed through retrograde urethrogram) as the probable cause of LUTO. This contradicts the $0.6 \%$ incidence rate recorded by Santucci et al. in 2007 [21] for a study on urethral strictures among males in Michigan, USA. In their study, $41 \%$ of their subjects also had lower UTI. The vast difference in the statistical finding figures in both studies could be attributed to race, ethnicity, genetics, etc.

In relation to the microbiology aspect of this study, microbial organisms such as E. coli, Klebsiella species, Pseudomonas species, among others, were isolated as the causative organisms of lower UTI in our study patients. This is consistent with a study done by Hidron et al., 
2008 [11], in the United States. Similar organisms found in these two studies could be attributed to similar conditions of the study subjects. Generally, these organisms are associated with UTI worldwide. The even distribution of the organisms among the various age groups and occupations suggests that age and occupation were not predetermining factors for UTI especially in patients with LUTO.

Again, E. coli, Klebsiella species, Pseudomonas species, Staphylococcus aureus as well as Proteus species that were isolated from the study subjects are very consistent with a catheter associated UTI study conducted by Taiwo et al., 2006 [12], in Nigeria. The similarity of organisms in these two studies could be attributed to similar conditions of the study subjects. Ghana and Nigeria are both West African countries and therefore other possible factors for obtaining similar results could be due to similar cultures and geographical settings.

\section{Conclusion}

The most common cause of lower urinary tract obstruction (LUTO) in Kumasi, Ghana is prostatic hyperplasia and the commonest causative organism of the associated UTI symptom is Escherichia coli.

\section{Acknowledgements}

We are thankful to all the study participants for taking part in the study. We are also thankful to the staff of the Urology and Microbiology Departments, KATH and KCCR for their support during the study.

\section{Authors' Contributions}

C. K. Gyasi-Sarpong planned and designed the study protocol, carried out the study and headed the writing of the manuscript. E. M. T. Yenli and A. Idriss supervised the study and contributed immensely to the writing of the manuscript. R. Azorliade, K. Aboah, A. A. Arhin, A. A. Annan and Y. A. Boaitey contributed to the study and writing of the manuscript. All the authors have read and approved the manuscript.

\section{REFERENCES}

[1] V. W. Nitti, "Pressure Flow Urodynamic Studies: The Gold Standard for Diagnosing Bladder Outlet Obstruction," Reviews in Urology, Vol. 7, Suppl. 6, 2005, pp. 14-21.

[2] C. Dawson and H. Whitfield, "ABC of Urology: Bladder Outflow Obstruction,” BMJ, Vol. 312, No. 7033, 1996, pp. 767-770. doi:10.1136/bmj.312.7033.767

[3] J. S. Lam, K. L. Cooper and S. A. Kaplan, "Changing Aspects in the Evaluation and Treatment of Patients with Benign Prostatic Hyperplasia," Medical Clinics of North
America, Vol. 88, No. 2, 2004, pp. 281-308. doi:10.1016/S0025-7125(03)00147-0

[4] A. J. Schaeffer and E. M. Schaeffer, "Infection of the Urinary Tract," In: A. J. Wein, et al., Eds, CampbellWalsh Urology, 9th Edition, Saunders, Philadelphia, 2007, pp. 223-303.

[5] E. H. Kass and M. Finland, "Asymptomatic Infections of the Urinary Tract," Transactions of the American Society of Physiology, Vol. 69, 1956, pp. 56-64.

[6] T. L. Griebling, "Urinary Tract Infection in Women," In: M. S. Litwin and C. S. Saigal, Eds., Urologic Diseases in America. US Department of Health and Human Services, Public Health Service, National Institutes of Health, $\mathrm{Na}$ tional Institute of Diabetes and Digestive and Kidney Diseases, US Government Printing Office: NIH Publication, Washington DC, 2007, pp. 589-619.

[7] J. W. Warren, "Catheter-Associated Urinary Tract Infection," Infectious Disease Clinics of North America, Vol. 11 , No. 3, 1997, pp. 609-622. doi:10.1016/S0891-5520(05)70376-7

[8] C. M. Kunin, "Urinary Tract Infections: Detection, Prevention, and Management," Williams \& Wilkins, Baltimore, 1997.

[9] S. M. Schappert, "Ambulatory Care Visits to Physician Offices, Hospital Outpatient Departments, and Emergency Departments: United States, 1997," Vital and Health Statistics Series 13, No. 143, 1999, pp. 1-39.

[10] T. L. Griebling, "Urinary Tract Infection in Men," In: M. S. Litwin and C. S. Saigal, Eds., Urologic Diseases in America. US Department of Health and Human Services, Public Health Service, National Institutes of Health, National Institute of Diabetes and Digestive and Kidney Diseases, US Government Printing Office: NIH Publication, Washington, 2007, pp. 623-645.

[11] A. L. Hidron, J. R. Edwards, J. Patel, et al., "NHSN Annual Update: Antimicrobial-Resistant Pathogens Associated with Healthcare-Associated Infections: Annual Summary of Data Reported to the National Healthcare Safety Network at the Centers for Disease Control and Prevention, 2006-2007," Infection Control and Hospital Epidemiology, Vol. 29, No. 11, 2008. doi:10.1086/591861

[12] S. S. Taiwo and A. O. A. Aderounmu, "Catheter Associated Urinary Tract Infection: Aetiologic Agents and Antimicrobial Susceptibility Pattern in Ladoke Akintola University Teaching Hospital, Osogbo, Nigeria," African Journal of Biomedical Research, Vol. 9, 2006, pp. 141148.

[13] C. Turpin, B. Minkah, K. Danso and E. Frimpong, “Asymptomatic Bacteriuria in Pregnant Women Attending Antenatal Clinic at Komfo Anokye Teaching Hospital, Kumasi, Ghana," Ghana Medical Journal, Vol. 41, No. 1, 2007, pp. 26-29.

[14] M. Cheesbrough, "Parasitological Tests," In: A. Moody, G. Malcolm and W. John, Eds., District Laboratory Practice in Tropical Countries, Vol. 1, Cambridge University Press, New York, 2009.

[15] R. W. Haley, D. H. Culver, J. W. White, M. W. Morgan and T. G. Emori, "The Nationwide Nosocomial Infection 
Rate: A New Need for Vital Statistics," American Journal of Epidemiology, Vol. 121, No. 2, 1985, pp. 159-167.

[16] J. P. Patton, D. B. Nash and E. Abrutyn, "Urinary Tract Infection: Economic Considerations," Medical Clinics of North America, Vol. 75, No. 2, 1991, pp. 495-513.

[17] W. E. Stamm and T. M. Hooton, "Management of Urinary Tract Infections in Adults," The New England Journal of Medicine, Vol. 329, No. 18, 1993, pp. 1328-1334. doi:10.1056/NEJM199310283291808

[18] H. C. Kuo, "Clinical Prostate Score for Diagnosis of Bladder Outlet Obstruction by Prostate Measurements and Uroflowmetry," Urology, Vol. 54, No. 1, 1999, pp.
90-96. doi:10.1016/S0090-4295(99)00092-8

[19] J. Isaac and D. S. Coffey, "Etiology and Disease Process Of Benign Prostatic Hyperplasia," Prostate, Vol. 2, 1987, pp. 33-50.

[20] D. M. Peehl, P. Cohen and R. G. Rosenfeld "The InsulinLike Growth Factor System in the Prostate," World Journal of Urology, Vol. 13, No. 5, 1995, pp. 306-311. doi:10.1007/BF00185974

[21] R. A. Santucci, G. F. Joyce and M. Wise, "Male Urethral Stricture Disease," The Journal of Urology, Vol. 177, No. 5, 2007, pp. 1667-1674. doi:10.1016/j.juro.2007.01.041 